\title{
A Cryogenically Cooled, Multidetector Spectrometer for Infrared Astronomy
}

Fred C. Witteborn and Jesse D. Bregman

\section{LIBRARY CQPY}

$$
p^{2}-1984
$$

LANGLEY RESEARCH CENTER LISRARY, NASA HAMPTON, VIRGINIA 


\section{A Cryogenically Cooled, Multidetector Spectrometer for Infrared Astronomy}

Fred C Witteborn,

Jesse D Bregman, Ames Research Center, Moffett Field, Calıfornı 
A cryogenically cooled, multidetector spectrometer for infrared astronomy

Fred C. Witteborn and Jesse D. Bregman

Space Science Division, NASA Ames Research Center

Moffett Field, California 94035

\section{Abstract}

A liquid-helium-cooled, 24-detector grating spectrometer has been developed and used for low-resolving-power astronomical observations in the 5-14-um spectral range. The instrument has operated on the $91-\mathrm{cm}$ Kulper Alrborne Observatory (KAO), the 3-m IRTF (Mauna Kea), the 3-m Shane telescope (Lick observatory), and the 152-cm NASA and University of Arizona telescope (Mt. Lemmon, Arlz.). The detectors are discrete $S_{1}: B_{2}$ photoconductors with individual metal oxide semiconductor field-effect transistor (MOSFET) preamplifiers operating at $4 \mathrm{~K}$. The system uses a liquid-helium-cooled slit, order-sorter filter, collimator mirror, gratıng, and camera mirror arranged in a Czerny-Turner configuration, with a cold stop added between the collimator mirror and the grating. The distances between components are chosen so that the collimator mirror images the telescope's secondary mirror onto the cold stop, thus providing a very effective baffle. Scattered radiation is effectively reduced by using liquid-helıum-cooled, black baffles to divide the spectrometer into three separate compartments. The system noise-equivalent flux density, when used on the 152-cm telescope from 8 to $13 \mu \mathrm{m}$ with a resolving power of $50,154.4 \times 10^{-17} \mathrm{Wcm}^{-2}{\mu \mathrm{m}^{-1}} \mathrm{~Hz}^{-1 / 2}$. The maln applications are for measuring continuum radiation levels and solid-state emission and absorption features in regions of star and planet formation.

\section{Introduction}

In astronomical research there is a continual effort to improve the sensitivity of the viewing instruments. This may be done elther by gathering more photons or by making more efficient use of the ones collected. The need for greater sensitivity is particularly evident at the infrared wavelengths, at which the most important diagnostic spectral features of molecules and solids appear, but at which only the brightest objects have been studied. In response to this need, a multiple-detector grating spectrometer for use in the 5-14-um micrometer range was bullt; it takes advantage of the greatly improved efficiency offered by simultaneously integrating at different wavelengths. Most earlier spectroscopic work in this wavelength range was done with circular, variable filter spectrometers with single detectors. A multiple-detector system with liquid-helium-cooled components was developed and demonstrated by Rank and Bregman. 1

The system described here uses similar electronics and data handling, but features improved detectors, optics, and internal baffling. So far the system has operated at resolving powers from 50 to 60 , but resolving powers up to 300 can be accommodated with appropriate gratings. The primary purpose of the instrument was to study dust-emission features that might be associated with regions of planet formation. It has, in fact, been applied to a much broader variety of objects ranging from comets to seyfert galaxies. Because of the strong emphasis on sensitivity inherent in its design, it is called the Falnt Object Grating spectrometer (FOGS). In this paper we describe briefly the design of the FOGS and then its performance characteristics when used with a ground-based telescope and with the Kulper Alrborne Observatory (KAO).

\section{Design}

The design of the FOGS was guided by the need for the highest possible sensitivity coupled with sufficient spectral resolving power to study the shapes of absorption features expected from silicates near $10 \mathrm{\mu m}$ and from water 1 ce near $6.3 \mathrm{um}$, and the shapes of emission features from silicates near $10 \mu \mathrm{m}$ and from silicon carbide near $11.3 \mu \mathrm{m}$. A resolving power of 50 is adequate for many purposes and permits study of the entire 8-13-um spectral "window," with 24 detectors at a single setting of an appropriate grating. This window can be studied from large, ground-based telescopes at sufficiently dry sites. Use of the KAO at an altitude of $13 \mathrm{~km}$ to get above most of the atmospheric water permits the same instrument, with a different grating, to disperse the 5-8-um spectral range across the same 24 detectors with a resolving power of 60 . The sensitivity was optimized through the cholce of detectors and minimization of stray infrared radiation.

The cholce of detectors was dictated by quantum efficlency, nolse performance, cost, and the ability to fabricate them in a package compatible with use in an existing spectrometer. (This latter condition was dictated by the desire to use some of the detectors before enough money was avallable to buy all the parts for a new spectrometer.) The most sultable 
detectors avallable without additional development costs were the discrete $S_{1}: B_{1}$ detectors bullt by Aerojet Electro Systems. Laboratory tests showed that their quantum efficiencies were between 0.25 and 0.4 (Yee, personal communication, 1979). Their nolse equivalent power is somewhat below $10^{-15} \mathrm{WHz}^{-1 / 2}$, which 15 smaller than fluctuation nolse in the background radiation anticipated in the 28 spectral bands to be viewed by individual detectors, as shown below.

The background radiation is dominated over much of the spectral range of interest by themal emission from the telescope optics and the Dewar window. Their combined emissivity $\varepsilon \quad 1 s$ about 0.3 and temperatures, $T$, are typlcally $280 \mathrm{~K}$. The spectrometer silt aperture was chosen to be round $(1.0 \mathrm{~mm} i n$ diameter) for the nominal design. This provides a 9-arcsec fleld of view (FOV) on the $\mathrm{f} / 17 \mathrm{Mt}$. Lemmon $152-\mathrm{cm}$ telescope and a $14-a r c s e c$ FOV on the KAO. The resulting background radiation power $P$ arriving at the detector at wavelength $L$ is, from the Planck radiation law,

$$
P=\varepsilon \pi D^{2} \Omega \Delta L c_{1} /\left\{4 L^{5}\left\{\exp \left(c_{2} / L T\right)-1\right]\right\}
$$

where $D$ is the diameter of the slit aperature; $\Omega$ is the solid angle of the acceptance cone, determined by the cold baffling in the spectrometer; $\Delta L$ is the spectral band arriving at a single detector; $c_{1} 1 \mathrm{~s} 3.74 \times 10^{-12} \mathrm{Wcm}^{2} ;$ and $c_{2} 1 \mathrm{~s} 1.438 \mathrm{~cm} \mathrm{deg}$. For $1 \mathrm{deal} \mathrm{cold}$ baffling in the spectrometer and an $f / 17$ system, $\Omega$ is 0.0027 sr. The resulting background power is $3.1 \times 10^{-9} \mathrm{~W}$ and the fluctuation noise power is $7.5 \times 10^{-15} \mathrm{WHz}^{-1 / 2}$. The backgrounds actualiy detected must be multiplied by the quantum efficiency $\eta$, and the fluctuation noise power detected by $\eta^{1 / 2}$. Consequently, the detected 5-um fluctuation norse power is $1.6 \times 10^{-15} \mathrm{WHz}^{-1 / 2}$, so the intrinsic detector nolse of $1 \times 10^{-15} \mathrm{WHz}^{-172} 1 \mathrm{~s}^{\mathrm{s}} \mathrm{sme-}$ what smaller than the smallest anticipated fluctuation noise power. The detectors have rectangular collecting surfaces that are $1.0 \mathrm{~mm}$ wide by $3.0 \mathrm{~mm}$ long. Width 15 measured along the direction of dispersion. The detectors were fabricated in groups of six, with center-to-center distances of $1.27 \mathrm{~mm}$. The overall detector packages, including sourcefollower preamplifiers are $7.62 \mathrm{~mm}$ wide, $20.0 \mathrm{~mm}$ long, and $6.5 \mathrm{~mm} \mathrm{hlgh}$. Four packages arranged along the direction of dispersion cover the wavelength range to be studied in a total dispersion distance of $30.5 \mathrm{~mm}$.

A Czerny-Turner optical configuration was chosen for ease of conversion from one wavelength range to another and for ease of baffling against stray radiation. The conversion from the 8-14-um range to the 5-8-um range requires only replacing the grating and an ordersorting filter. The gratings are used in first-order and are chosen to give reasonably high efficiencies (508) in both polarizations over the range of operation. The grating used for 8-13-um work is a Bausch and Lomb replica on borosilicate Crown glass with $50 \mathrm{grooves} / \mathrm{mm}$, and a blaze angle of $13^{\circ}$. When used with the optical components and spacings given $1 \mathrm{n}$ Table 1, this grating provides a spectral resolution of $0.22 \mu \mathrm{m}$ between detector elements. The grating used for 5-8-um work is similar, but with 90 grooves/mm and a blaze of $17.45^{\circ}$, providing a spectral resolution of $0.11 \mathrm{\mu m}$ between detector elements.

Table 1. Optical parameters

\begin{tabular}{lccc}
\hline \multicolumn{1}{c}{ Optical Element } & $\begin{array}{c}\text { Size, } \\
\mathrm{mm}\end{array}$ & $\begin{array}{c}\text { Focal length, } \\
\mathrm{mm}\end{array}$ & $\begin{array}{c}\text { Distance from Preceding } \\
\text { Optical Element, } \mathrm{mm}\end{array}$ \\
\hline $\begin{array}{l}\text { Slit aperture, diameter } \\
\text { Colizmator mirror, diameter }\end{array}$ & 1 & -- & -- \\
$\quad$ (parabola) & 13 & 120 & 120 \\
Pupil, diameter & 10 & -- & 120 \\
Grating & $26 \times 26$ & flat & 160 \\
Camera mirror (sphere) & $20 \times 60$ & 125 & 125 \\
Bank of 24 Fabry mirrors & $1.27 \times 3.0$ & 8.1 & 7.7 \\
Detectors & $1.0 \times 3.0$ & -- & \\
\hline
\end{tabular}

A schematic of the spectrometer, including the optical layout, is shown in Figure 1. light enters through a potassium bromide window at the Dewar bottom and strikes a $45^{\circ}$ flat that directs the beam to the entrance (or slit) aperture and order-sorting filter. An important feature for proper baffling is the placement of the collimator mirror so that it images the telescope secondary onto a black, cold stop (the pupil). Light from off-axis sources consequently cannot pass beyond the pupll unless it scatters off of one of the telescope mirrors into the main optical beam. Furthermore, each component that must dissipate off-axis or out-of-band radiation 15 surrounded by a blackened chamber, lighttight expect along the optical axis, so that scattered radiation cannot easily reach the detectors. Zeroth-order radiation from the grating strikes a blackened panel not directly viewed by the detectors; $3 M$ Nextel Velvet was used as the blackening agent. The dispersed 


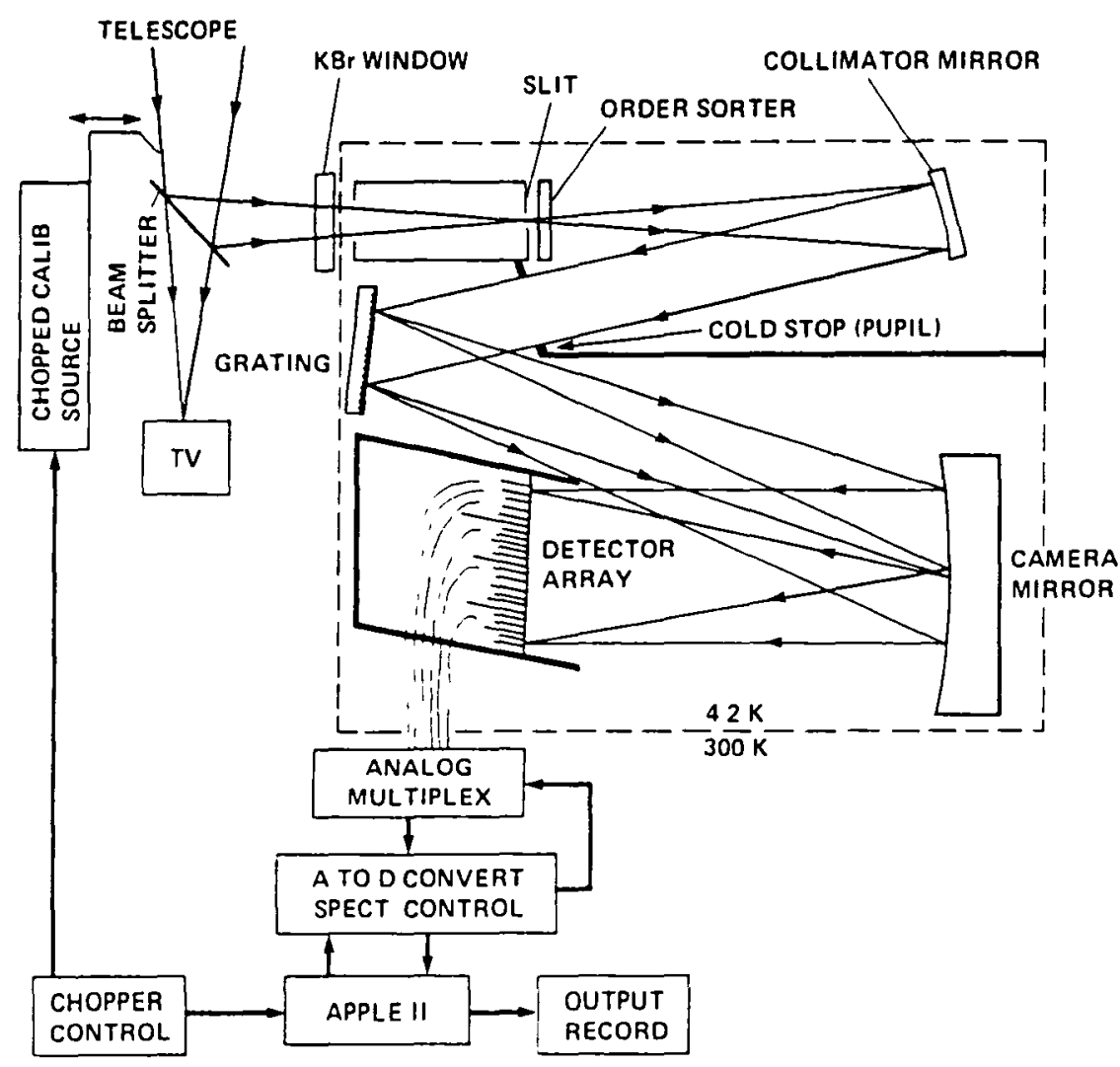

Figure 1. Schematic drawing of the Faint object Grating Spectrometer (FOGS). The 24 detectors are SI.B1 photoconductors. The 24 individual Fabry mirrors are not shown separately since they are part of the detector assembly. The beam splitter is a thin gold film that reflects nearly all infrared radiation, but allows 508 of the visible light to reach a TV camera, parfocal with the slit. This permits guiding on the object whose spectrum is being taken. The chopped calibration source may be moved into the field of view when needed. radiation from the grating is focused by the camera mirror onto 24 adjacent Fabry mirrors which form 24 images (at different wavelengths) of the pupil onto detectors facing them. The use of the Fabry mirrors ensures that radiation in the telescope's light cone arriving at any part of the slit aperture will be spread over the same spot on the detector. This reduces errors in wavelength and intensity caused by the interaction of image motion across the aperture and varlations in quantum efficlency across individual detectors. Fabrication of the multiple Fabry optics was modeled after the technique used by Erickson (personal communication, 1982).

The glass optical components are mounted in Invar frames which are held, in turn, to aluminum frames by spring-loaded screwadjustment mechanisms. The aluminum frames are bolted to an aluminum optical bench which is bolted to the $20-\mathrm{cm}-$ diam copper bottom-plate of a Dewar (Infrared Laboratorles, catalog No. HD3-8, w1th extended length). Compliance for thermal expansion between the aluminum and copper and between the aluminum and Invar interfaces is provided by the screws. Thermal contact between the glass and the optical bench is provided by copper straps

bolted to the bench and glued with rubber cement ${ }^{2}$ to the backs of the mirrors. The entire optical and detector assembly is enclosed by blackened aluminum surfaces held at a temperature near $4.2 \mathrm{~K}$. The optical components may be cooled from $77 \mathrm{~K}$ to $4.2 \mathrm{~K}$ in about $1 \mathrm{hr}$. cooling from room temperature to $77 \mathrm{~K}$ takes 3 to $5 \mathrm{hr}$. At present, there are no moving parts in the spectrometer. All alignment is done before the Dewar is closed up. Wavelength selection must also be done before the Dewar is closed. This is accomplished by alming a helium-neon laser beam through the spectrometer (w1th the order-sorter filter removed). A high-order image nearest the blaze angle will be brightest and may be used to set the grating angle. After pump-out and cool-down, the settıng can be checked against a known calibration standard, such as an ammonia absorption cell.

Signals from the $S_{1}: B_{1}$ photoconductor detectors are conditioned for transmission out of the Dewar by adjacent source-follower amplifiers (using specially selected 3N163 MOSFETs) operating at the detector temperatures, near $4 \mathrm{~K}$. The load resistors are $3 \times 10^{\circ}$ ohm dies. A requlated power supply maintains a common drain voltage of $-10 \mathrm{~V}$ and an adjustable, positive $(0$ to $10 \mathrm{~V})$, common detector bias. Each amplifier output is further amplified by a warm (external to the Dewar) amplifier which also serves to integrate the resultant signal with a time-constant longer than the sampling time, but shorter than the optical modulation (chopping) time. The signals, in turn, are sampled by an analog multiplexer, further amplified, digitized, and finally stored by the computer controller. In this way, phasesensitive detection is done digitally by the computer. 


\section{Operation}

The spectrometer is operated at the focal plane of an astronomical telescope equipped with a movable secondary or tertiary mirror (called the chopper). The purpose of moving the mirror 13 to place, alternately, the object-plus-background radiation and then the background radiation alone on the detectors. The resulting difference in signal is a measure of radiation from the object alone. The computer initiates a sampling sequence of the 24 integrated outputs in phase with the chopper. All detectors are sampled several times after each motion of the chopper mirror. The data storage program automatically stores signals from opposite ends of the travel of the mirror for each detector and then subtracts the sums sequentially. As the sequence of detector interrogations is repeated, the new data are added to those already stored. After a preset period of data accumulation, the results are presented on a TV screen; they may also be stored on a magnetic disk. The position of each detector corresponds to a wavelength, which may be calibrated by comparison with absorption spectra taken through gas cells (typlcally ammonia or methane) or through polystyrene. Fluxes are determined by comparisons with spectra of bright, standard stars. Relative spectral response is established by comparison with a laboratory blackbody. The wavelength calibrations are made at least once during each night of observing. Comparisons with standard stars are made several times during each night of observing to provide not only flux comparisons, but also a means of correcting for the atmospheric absorption spectrum.

\section{Performance}

Observations made with the FOGS on the Mt. Lemmon 152-cm telescope produced spectra with one-sigma errors in individual channels $\left(0.22-\mu \mathrm{m}\right.$ resolution) of about $1 \times 10^{-18} \mathrm{Wcm}^{-2} \mu \mathrm{m}^{-1}$ in 30 min near $8.4 \mathrm{\mu m}$. This corresponds to a system nolse-equivalent flux density (NEFD) of $4.7 \times 10^{-17} \mathrm{Wcm}^{-2} \mathrm{~Hz}^{-1 / 2}$. On the KAO, the FOGS uses the same $\mathrm{f} / 17$ cone. When operating with the same slit aperture diameter of $1 \mathrm{~mm}$, with a resolution of $0.11 \mathrm{um}$ at $6.2 \mu \mathrm{m}$, the background is lower. The signal is reduced by both the bandwidth and the aperture reduction $(91 \mathrm{~cm}$ for KAO versus 152 for $\mathrm{Mt}$. Lemmon). The resulting system NEFD for FOGS on the KAO is $1.4 \times 10^{-16} \mathrm{Wcm}^{-2} \mathrm{um}^{-1} \mathrm{~Hz}^{-1} / 2$ at $6.2 \mathrm{\mu m}$. In $30 \mathrm{~min}$ of integration, a one-sigma error of about $3 \times 10^{-18} \mathrm{Wcm}^{-2} \mathrm{\mu m}^{-1} \mathrm{can}$ be obtained. Since this performance is being attained simultaneously in 24 channels, the desired spectrum can be obtained much faster than with a single detector instrument, even if it has a somewhat better detector.

The use of a 24-detector system covering the entire band-pass of interest has two 1 mportant advantages over scanning, single-detector systems. First, if the quantum efficiencies were comparable, the proposed system would be 24 times faster in obtaining spectra of comparable quality. Actually, since the quantum efficiencies of the $S_{1}: B_{1}$ detectors average to only half that of the best single detector used at $6 \mu \mathrm{m}$, the advantage in speed is reduced to a factor of 6 -still a very important advantage. Second, the multiple-detector system is relatively insensitive to temporary polnting errors and variations in seeing conditions, which can cause erroneous spectral features in scanning systems.

Although the FoGs is relatively simple and convenient to use, care must be exercised in ensuring that the object is centered on the slit. When a spectrum $A_{1}$ of an astronomical object $A$ has been obtained, it is typically divided by a spectrum $S_{1}$ of a bright standard star $S$ obtained through a similar alr mass. The quotient is then multiplied by the known (through theoretical extrapolation or previous measurement) spectrum $Q_{1}$ of the standard. The resulting spectrum

$$
F_{1}=A_{1} Q_{1} / S_{1}
$$

represents our determination of the actual spectrum of the astronomical object. This procedure can lead to an error if there are systematic differences in the pointing of the telescope on object $A$ and standard $S$. The error arises because a shift in star position at the slit aperture appears as a shift in the spectrum on the Fabry array. Consequently, a deep atmospheric feature in $A_{1}$ and $S_{1}$ is not properly corrected when one is divided by the other. Tests at the $M t$. Lemmon $152 \mathrm{~cm}$ telescope using a 1 -mm aperture showed that polnting errors of up to $0.2 \mathrm{~mm}$ in any direction produced negligible errors in the 8-13-um range, which includes the deep, sharp ozone-absorption feature. Polnting errors of $0.5 \mathrm{~mm}$ produced a serious spectral distortion (308 in several channels), however. Differential refraction causes a separation between the visual images used for guiding and the $1 \mathrm{nfrared}$ image when observing at large angles from the zenith. This effect coupled with a small amount of flexure in the spectrometer produces errors in boresight of up to $0.2 \mathrm{~mm}$. Such errors are readily avolded by reboresighting on a bright object in the part of the sky under study. 
Some spectra obtained with the FoGs are shown in Figures 2-5. Figure 2 is a spectrum of Alpha Orionis which exhibits a well-known, bright, silicate emission feature. Figure 3 is a spectrum of AFCRL 2232 showing 1 ts broad silicon carbide emission feature. Figure 4 is a

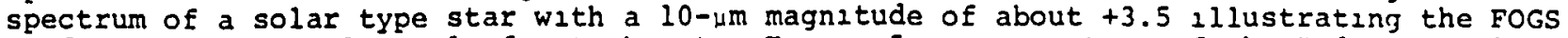
performance on a relatively faint object. Figure 5 is a spectrum of the Red Rectangle (HD 44179) taken from the KAO with a 20-arcsec beam diameter. Emission features believed associated with dust are clearly defined at 6.2 and $7.7 \mathrm{um}$. A number of FoGs spectra have already been used in published articles. These include an 8-13-um spectrum of the Seyfert galaxy Markarian 231 (Reference 4), 5-8-um spectra of the reflection nebulae, NGC 7023 and NGC 2023 (Reference 5), 5-8-um spectra of absorption features in reglons of star formation, ${ }^{6}$ and 8-13-um spectra of asterolds ${ }^{3}$ and Comet IRAS-Arak1-Alcock. ${ }^{8}$ Additional articles on FoGs spectra are in press or in preparation.

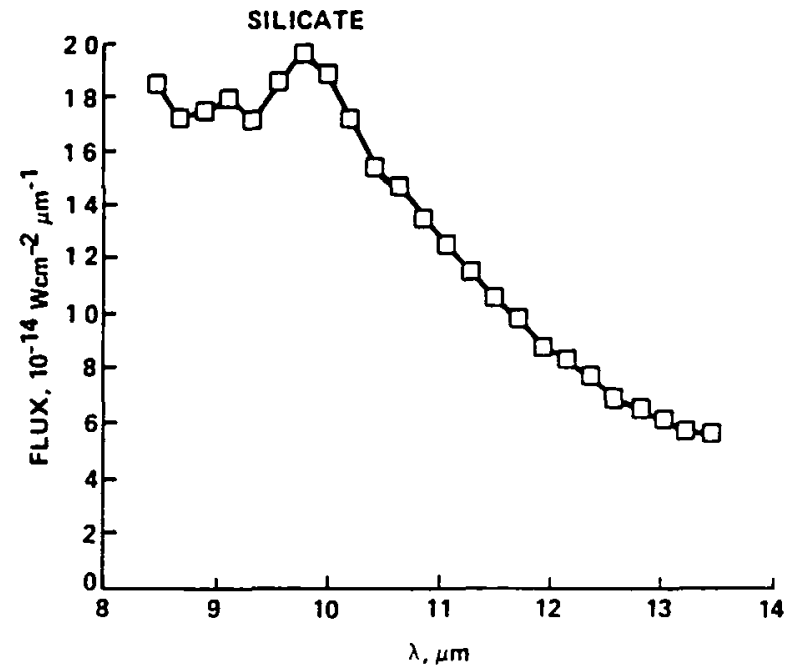

Figure 2. A spectrum of Alpha Orionis showing its prominent silicate emission feature which arises from circumstellar dust.

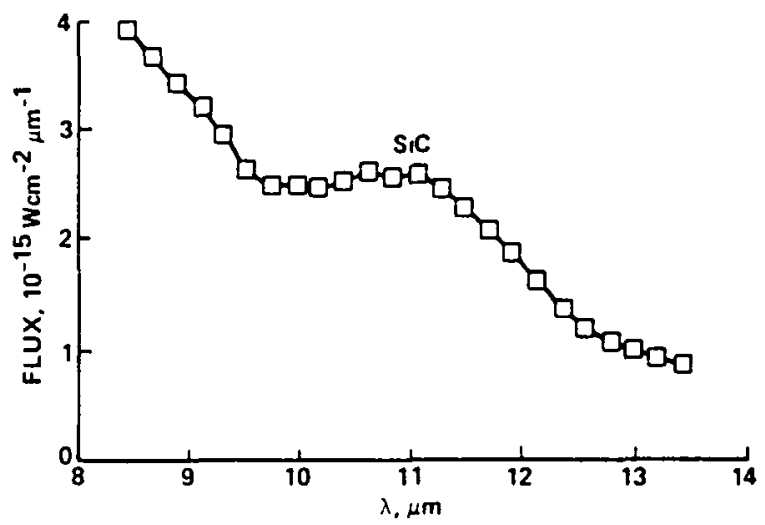

Figure 3. A spectrum of the carbon star AFCRL 2232 showing the broad emission feature attributed to circumstellar silicon carbide.

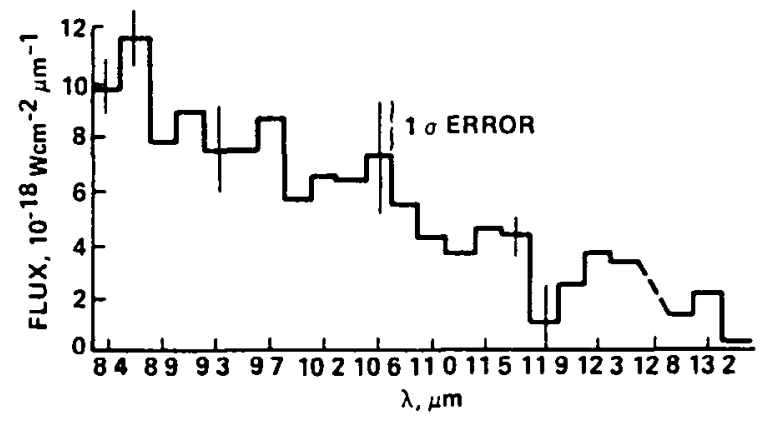

Figure 4. A spectrum of Eta Coronae Borealis, a G2V star with visual magnitude +4.9 . Integration time was $32 \mathrm{~min}$, using the Mt. Lemmon 152-cm telescope.

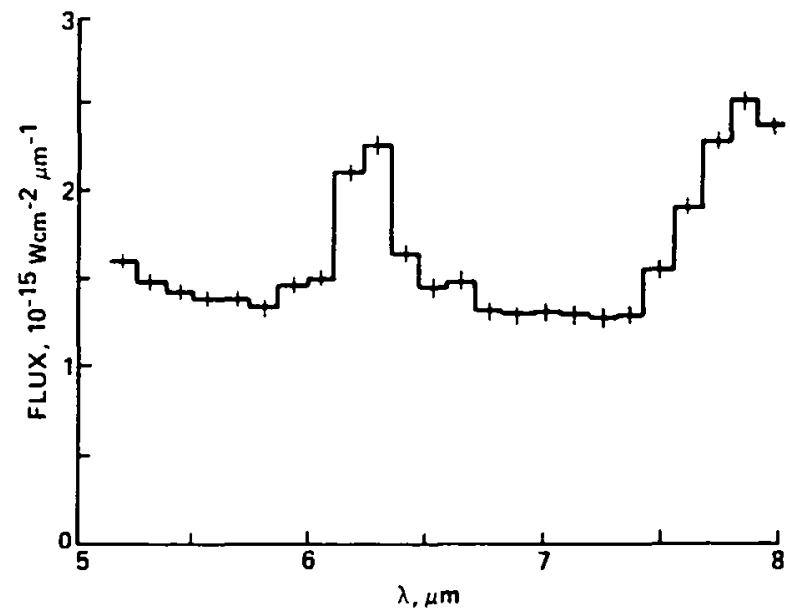

Figure 5. A spectrum of HD44179 (the Red Rectangle) obtained from the KAO with $12 \mathrm{~m} n$ of integration. The unknown dust emission features near 6.2 and $7.7 \mathrm{~km}$ are prominent. 


\section{Concluding Remarks}

The anticipated improvements in efficiency stemming from the use of multiple-detector systems have been realized in the FoGs. The spectra are obtained with about 6 times the efficiency of the best single-detector scanning devices and some of the problems inherent in slow, sequential spectral sampling are avolded. The limiting sensitivity is about one magnitude fainter than for the best single-detector devices for obtaining spectra at comparable resolving powers in comparable integration times.

\section{Acknowledgments}

It is a pleasure to thank D. Rank for the use of electronics from his spectrometer; H. Crean for fabrication of the FOGS mechanical parts; the staff of the NASA and University of Arizona $152-\mathrm{cm}$ telescope at Mt. Lemmon for many successful and efficient observing runs; and the staff of the Kulper Alrborne Observatory for the successful lnaugural flights of the FOGS.

\section{References}

1. Rank, D. M. and Bregman, J. D., "Multıchannel Spectrometers for Infrared Astronomy," Bull. Am. Astron. Soc., Vol. 7, p. $409,1975$.

2. O'Brien, K. and Witteborn, F. C., "Thermal Contacts between Metal and Glass for Use at CryogenlC Temperatures," NASA TM-85856, 1984.

3. Bregman, J. D., Observation and Interpretation of the Infrared Spectra of Bright Compact Nebulae, Ph.D. dissertation, U. of California, Santa Cruz, Calıf., 1976.

4. Bregman, J. D. and Witteborn, F. C., "The Unusual 8-13 Micron Spectrum of Markarıan 231," Astrophys. J. Lett., Vol. 281, Pp. L17-L19, 1984.

5. Sellgren, K., Allamendola, L. J., Bregman, J. D., werner, M. W., and Wooden, D. H., "The Four-Thirteen-Micron Spectrum of Reflection Nebulae, NGC 7023 and NGC 2023," Astrophys. J., in press, 1984.

6. Tlelens, A. G. G. M., Allamandola, L. J., Bregman, J. D., Goebel, J. H. , d'Hendecourt, L., and Witteborn, F. C., "Absorption Features in the Five-to-Eight Micron Spectra of Protostars," Astrophys. J., in press, 1984.

7. Felerberg, M. A., Witteborn, F. C., and Lebofsky, L. A., "A Survey of the 8 to 13

Micron Spectra of Maln Belt Astero1ds," Icarus, Vol. 56, pp. 393-397, 1983.

8. Felerberg, M. A., Witteborn, F. C., Campins, H. , and Johnson, J. R., "8 to 13 Micron Spectroscopy of Comet 1983d" (Abstract), Bull. Am. Astron. Soc., Vol. 15, p. 802, 1983. 


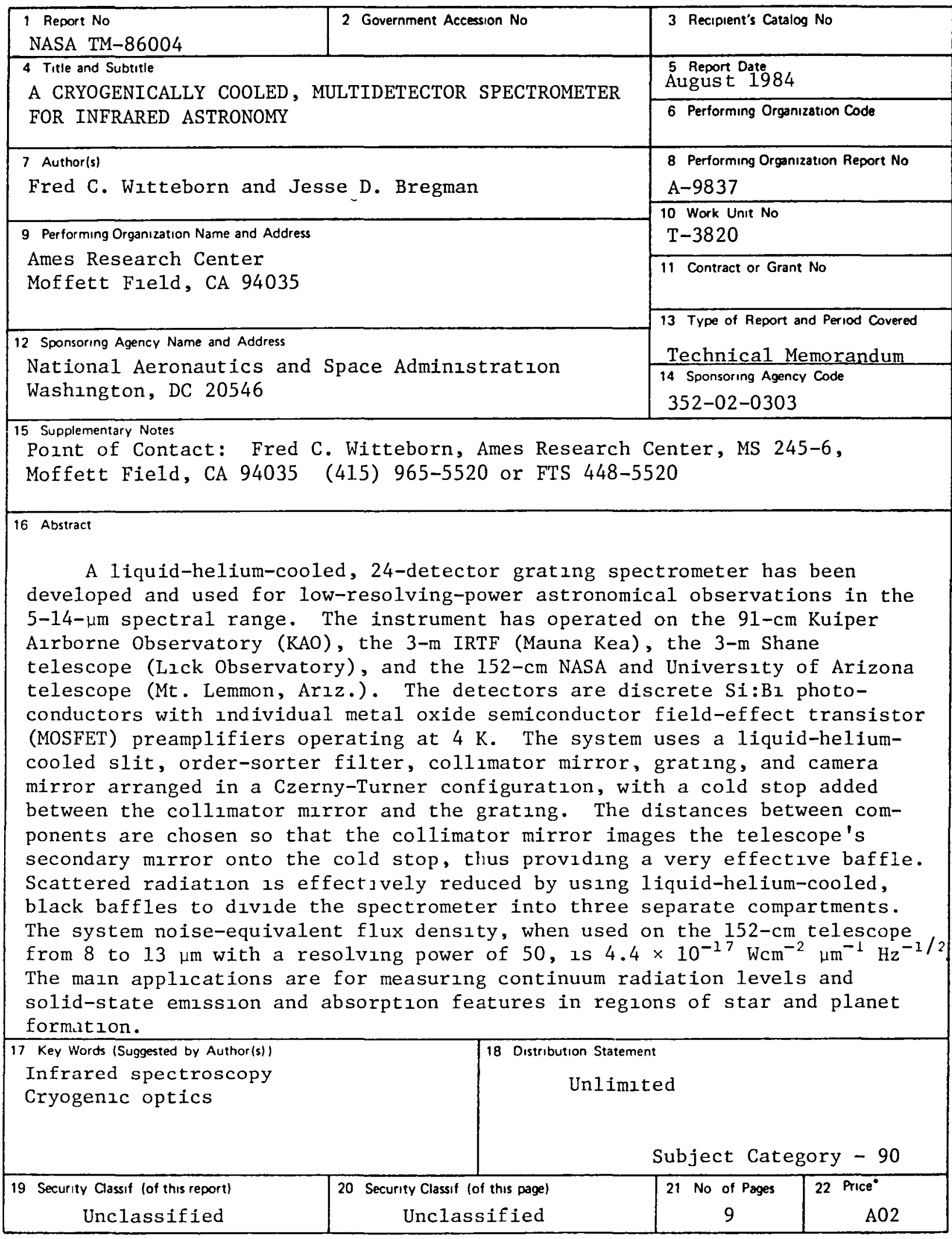

- For sale by the Natıonal Technical Information Service Soringfield, Virginia 22161 


\section{End of Document}

